\title{
COMPACT HILBERT CUBE MANIFOLDS AND THE INVARIANCE OF WHITEHEAD TORSION
}

\author{
BY T. A. CHAPMAN ${ }^{1}$ \\ Communicated by R. D. Anderson, June 16, 1972
}

\begin{abstract}
In this note we prove that every compact metric manifold modeled on the Hilbert cube $Q$ is homeomorphic to $|K| \times Q$, for some finite simplicial complex $K$. We also announce an affirmative answer to the question concerning the topological invariance of Whitehead torsion for compact, connected CW-complexes. As a corollary of this latter result it follows that two compact Hilbert cube manifolds are homeomorphic iff their associated polyhedra (in the sense above) have the same simple homotopy type.
\end{abstract}

1. Introduction. In this note we announce some recent results on infinite-dimensional manifolds which imply, among other things, the topological invariance of Whitehead torsion for compact connected CWcomplexes.

A Hilbert cube manifold (or $Q$-manifold) is a separable metric space which has an open cover by sets which are homeomorphic to open subsets of the Hilbert cube $Q$. We say that a $Q$-manifold $X$ can be triangulated (or is triangulable) provided that $X$ is homeomorphic ( $\cong$ ) to $|K| \times Q$, for some countable locally-finite simplicial complex $K$. In [5] it was shown that (1) any open subset of $Q$ is triangulable, and (2) if $X$ is any $Q$-manifold, then $X \times[0,1)$ is openly embeddable in $Q$ and thus is triangulable (where $[0,1)$ is the half-open interval). We refer the reader to [4] for a list of earlier results on $Q$-manifolds. In this note, based on results in [6], we prove that every compact $Q$-manifold can be triangulated. The triangulation of noncompact $Q$-manifolds is much more delicate and is expected to be the subject of a future paper.

Triangulation THEOREM. Every compact Q-manifold can be triangulated.

Using a result of West [13] it follows that if $K$ is any finite simplicial complex, then $|K| \times Q \cong M \times Q$, for some finite-dimensional combinatorial manifold $M$. In this sense it follows that all compact $Q$-manifolds can be combinatorially triangulated.

COROLLARY 1. Every compact Q-manifold can be combinatorially triangulated.

AMS (MOS) subject classifications (1969). Primary 5755; Secondary 5701.

Kev words and phrases. Hilbert cube manifolds, triangulation, Whitehead torsion, simple homotopy type, Property Z.

${ }^{1}$ Supported in part by NSF grant GP-28374. 
This result contrasts sharply with the corresponding finite-dimensional situation, as there exist compact finite-dimensional manifolds which cannot be combinatorially triangulated $[\mathbf{8}]$.

In [13] West proved that if $K$ is any finite complex, simplicial or CW, then $|K| \times Q$ is a $Q$-manifold. Combining this result with the Triangulation Theorem we have the following result on finiteness of homotopy types.

COROLlary 2. If $X$ is a compact metric space which is locally triangulable, then $X$ has the homotopy type of a finite complex.

In particular $X$ might be a compact $n$-manifold. Thus Corollary 2 strengthens the result of Kirby-Siebenmann on the finiteness of homotopy types of compact $n$-manifolds [9], but it is questionable whether this gives a simpler proof. It also sheds some light on the more general open question concerning the finiteness of homotopy types of compact ANR's [12].

The following result gives a topological characterization of simple homotopy types of finite $\mathrm{CW}$-complexes in terms of homeomorphisms on $Q$-manifolds. We refer the reader to [7] for a proof.

ChARACTERIZATION OF SIMPLE HOMOTOPY TYPES. Let $K, L$ be finite, connected $\mathrm{CW}$-complexes and let $f:|K| \rightarrow|L|$ be a map. Then $f$ is a simple homotopy equivalence iff the map

$$
f \times \mathrm{id}:|K| \times Q \rightarrow|L| \times Q
$$

is homotopic to a homomorphism of $|K| \times Q$ onto $|L| \times Q$.

We remark that the "only if" part of this theorem is just West's theorem on simple homotopy types [13]; i.e., if $f:|K| \rightarrow|L|$ is a simple homotopy equivalence, then $f \times \mathrm{id}:|K| \times Q \rightarrow|L| \times Q$ is homotopic to a homeomorphism. As an immediate corollary of the above theorem we answer affirmatively the question on the topological invariance of Whitehead torsion [11, p. 378].

Waldhausen is reported to have an earlier and completely different proof of this Whitehead invariance problem.

COROLlary 3. If $K, L$ are finite, connected $\mathrm{CW}$-complexes and $f:|K| \rightarrow$ $|L|$ is a homeomorphism, then $f$ is a simple homotopy equivalence.

We can also easily use the above Characterization to classify homeomorphic compact, connected $Q$-manifolds by simple homotopy type.

Corollary 4. Let $X, Y$ be compact, connected $Q$-manifolds and let

$$
X \cong|K| \times Q, \quad Y \cong|L| \times Q
$$

be any triangulations. Then $X \cong Y$ iff $|K|$ and $|L|$ have the same simple homotopy type.

Our proof we give here of the Triangulation Theorem and the proof of 
the Characterization of Simple Homotopy Types, which is given in [7], rely mainly on the work in [6]. For our purposes here we use the following result from [6].

For notation $R^{n}$ denotes Euclidean $n$-space, $B^{n}$ denotes the standard $n$-ball of radius $1, \operatorname{Int}\left(B^{n}\right)$ denotes the interior of $B^{n}$, and $S^{n-1}$ denotes the boundary.

StRAightening Lemma. If $X$ is a triangulated $Q$-manifold and $h: R^{n} \times Q \rightarrow X$ is an open embedding, for $n \geqq 2$, then $X \backslash h\left(\operatorname{Int}\left(B^{n}\right) \times Q\right)$ is a triangulated $Q$-manifold.

The proof of the Straightening Lemma given in [6] uses a considerable amount of infinite-dimensional machinery - influenced by finite-dimensional techniques. Some of the techniques used are infinite-dimensional surgery and infinite-dimensional handle straightening. These are just infinite-dimensional versions of some finite-dimensional techniques which were used in [9] for the recent triangulation results concerning $n$-manifolds.

Doing surgery on $Q$-manifolds is not nearly as difficult as it is on $n$-manifolds. In particular, the delicate inductive process of exchanging handles can always be done in two steps. Poincaré duality and transversality are never used. For straightening handles in $Q$-manifolds homeomorphisms on $T^{n} \times Q$ (where $T^{n}$ represents the $n$-torus) are used, much in the same way that torus homeomorphisms are used in finite-dimensional handle straightening. Also the theorem of West on simple homotopy type plays the role of the $s$-cobordism theorem.

In $\S 2$ we briefly describe some infinite-dimensional results which will be needed. Then in $\$ 3$ we use these results, along with the Straightening Lemma, to prove the Triangulation Theorem. We remark that no prior experience with infinite-dimensional topology is needed for reading this note.

2. Infinite-dimensional preliminaries. We view $Q$ as the countable infinite product of closed intervals $[-1,1]$ and we let 0 represent the point $(0,0, \ldots)$ of $Q$. Most basic is Anderson's notion of Property $\mathrm{Z}[\mathbf{1}]$. A closed subset $A$ of a space $X$ is said to be a $Z$-set in $X$ provided that for each nonnull and homotopically trivial open subset $U$ of $X, U \backslash A$ is also nonnull and homotopically trivial. A map $f: X \rightarrow Y$ (i.e., a continuous function) is said to be a $Z$-embedding provided that $f$ is a homeomorphism of $X$ onto a $Z$-set in $Y$. We now state two properties of $Z$-sets in $Q$-manifolds which will be needed in $\S 3$. For details see [2] and [5].

Approximation Lemma. Let $A$ be a compact metric space, $X$ be a $Q$-manifold, and let $f: A \rightarrow X$ be a map. Then $f$ is homotopic to a $Z$-embedding $g: A \rightarrow X$. In fact, $g$ can be chosen arbitrarily close to $f$. 
Collaring Lemma. Let $K$ be a finite complex, $X$ be a $Q$-manifold, and let $f:|K| \times Q \rightarrow X$ be a Z-embedding. Then there exists an open embedding $g:|K| \times Q \times[0,1) \rightarrow X$ such that $g(k, q, 0)=f(k, q)$, for all $(k, q) \in|K|$ $\times Q$.

We will also need the following result on the characterization of $Q$. For details see [5].

Characterization of $Q$. If $X$ is a compact contractible $Q$-manifold, then $X \cong Q$.

3. Proof of the Triangulation Theorem. Without loss of generality, let us consider a compact connected $Q$-manifold $X$ which we describe to triangulate. Since $X$ is a compact ANR (metric), it follows that $X$ is dominated by a finite complex (see [3, p. 106]). Thus $\pi_{1}(X)$ and $H_{*}(X)$ are finitely generated, where we use singular homology with integral coefficients. By a standard process, all of the homotopy groups of $X$ can be killed by attaching a finite number of $n$-cells to $X$, for $n \geqq 2$. One uses the fact that $\pi_{1}(X)$ is finitely generated to kill $\pi_{1}(X)$, and after this the Hurewicz isomorphism theorem is used to inductively kill the higher homotopy groups. Since $H_{*}(X)$ is finitely generated the process terminates after a finite number of cell attachments. We omit the details. Thus we obtain a finite sequence $X=X_{0}, X_{1}, \ldots, X_{p}$ of spaces such that each $X_{i}$ is obtained by attaching some $n$-cell to $X_{i-1}$, and $\pi_{n}\left(X_{p}\right)=0$ for all $n$. Since $X_{p}$ is an ANR, it must be contractible (see [3, p. 124]). Of course the spaces $X_{i}$, $1 \leqq i \leqq p$, are not $Q$-manifolds. We are going to modify the above procedure to obtain a sequence $X=X_{0}^{\prime}, X_{1}^{\prime}, \ldots, X_{p}^{\prime}$ of compact $Q$-manifolds such that each $X_{i}^{\prime}$ has the homotopy type of $X_{i}$. We construct this sequence inductively.

For each $j, 0 \leqq j \leqq p$, let $S_{j}$ be the following statement: There exists a sequence $X=X_{0}^{\prime}, X_{1}^{\prime}, \ldots, X_{j}^{\prime}$ of compact $Q$-manifolds such that (1) for $0 \leqq i \leqq j, X_{i}^{\prime}$ has the homotopy type of $X_{i}$, and (2) for $1 \leqq i \leqq j$, if $X_{i}^{\prime}$ is triangulable, then so is $X_{i-1}^{\prime}$. It is clear that $S_{0}$ is true. Thus assume that $S_{j}$ is true, for some $j<n$, and let $X=X_{0}^{\prime}, X_{1}^{\prime}, \ldots, X_{j}^{\prime}$ be the corresponding sequence of $Q$-manifolds satisfying (1) and (2). Let $\alpha: X_{j} \rightarrow X_{j}^{\prime}$ be a homotopy equivalence and let $f: S^{n-1} \rightarrow X_{j}$ be the attaching map used to construct $X_{j+1}$; i.e., $X_{j+1}=X_{j} \cup{ }_{f} B^{n}$. Define $f^{\prime}: S^{n-1} \times Q \rightarrow X_{j}^{\prime}$ by $f^{\prime}(x, q)=\alpha f(x)$, for all $(x, q) \in S^{n-1} \times Q$, and using the Approximation Lemma let $g: S^{n-1} \times Q \rightarrow X_{j}^{\prime}$ be a $Z$-embedding which is homotopic to $f^{\prime}$. We define $X_{j+1}^{\prime}=X_{j}^{\prime} \cup_{g}\left(B^{n} \times Q\right)$, the space formed by attaching $B^{n} \times Q$ to $X_{j}^{\prime}$. It easily follows from the Collaring Lemma that $X_{j+1}^{\prime}$ is a $Q$-manifold. In fact, there exists an open embedding $h: R^{n} \times Q \rightarrow X_{j+1}^{\prime}$ such that $X_{j}^{\prime} \cong X_{j+1}^{\prime} \backslash h\left(\operatorname{Int}\left(B^{n}\right) \times Q\right)$. Applying the Straightening Lemma it follows that if $X_{j+1}^{\prime}$ is triangulable, then so is $X_{j}^{\prime}$. All we need to do now 
is check that $X_{j+1}^{\prime}$ and $X_{j+1}$ have the same homotopy type. Let $g^{\prime}: S^{n-1} \rightarrow$ $X_{j}^{\prime}$ be defined by $g^{\prime}(x)=g(x, 0)$, for all $x \in S^{n-1}$. Since $Q$ contracts to $0 \in Q$, it follows that $X_{j+1}^{\prime}$ has the homotopy of $X_{j}^{\prime} \cup_{g^{\prime}} B^{n}$. But $g^{\prime}$ is homotopic to $\alpha f: S^{n-1} \rightarrow X_{j}^{\prime}$. Using Theorem 2.3 on p. 120 of $[\mathbf{1 0}]$, it follows that $X_{j}^{\prime} \cup_{g^{\prime}} B^{n}$ has the homotopy type of $X_{j+1}$. Thus $S_{j+1}$ is true.

Since $S_{p}$ is true we have a sequence $X=X_{0}^{\prime}, X_{1}^{\prime}, \ldots, X_{p}^{\prime}$ of compact $Q$-manifolds such that $X_{p}^{\prime}$ is contractible and if $X_{i}^{\prime}$ is triangulable, then so is $X_{i-1}^{\prime}$, for $1 \leqq i \leqq p$. Using the Characterization of $Q$ it follows that $X_{p}^{\prime} \cong Q$, which is triangulable. Then inductively working our way back down to $X$ it follows that $X$ is triangulable.

\section{REFERENCES}

1. R. D. Anderson, On topological infinite deficiency, Michigan Math. J. 14 (1967), 365-383. MR 35 \# 4893.

2. R. D. Anderson and T. A. Chapman, Extending homeomorphisms to Hilbert cube manifolds, Pacific J. Math. 38 (1971), 281-293.

3. K. Borsuk, Theory of retracts, Monografie Mat., Tom. 44, PWN, Warsaw, 1967. MR 35 \# 7306.

4. T. A. Chapman, Hilbert cube manifolds, Bull. Amer. Math. Soc. 76 (1970), 1326-1330.

5. - On the structure of Hilbert cube manifolds, Composito Math. (to appear).

6. - Surgery and handle straightening in Hilbert cube manifolds, Pacific J. Math. (submitted).

7. - Topological invariance of Whitehead torsion, Amer. J. Math. (submitted).

8. R. C. Kirby and L. C. Siebenmann, For manifolds the Hauptvermutung and the triangulation conjecture are false, Notices Amer. Math. Soc. 16 (1969), 695. Abstract \# 69T-G80.

9. - On the triangulation of manifolds and the Hauptvermutung, Bull. Amer. Math. Soc. 75 (1969), 742-749. MR 39 \#3500.

10. A. Lundell and S. Weingram, The topology of CW-complexes, Van Nostrand Reinhold, New York, 1969.

11. J. Milnor, Whitehead torsion, Bull. Amer. Math. Soc. 72 (1966), 358-426.

12. L. C. Siebenmann, On the homotopy type of compact topological manifolds, Bull. Amer. Math. Soc. 74 (1968), 738-742.

13. J. E. West, Mapping cylinders of Hilbert cube factors, General Topology and its Applications 1 (1971), 111-125.

Department of Mathematics, University of Kentucky, Lexington, Kentucky 40506. 